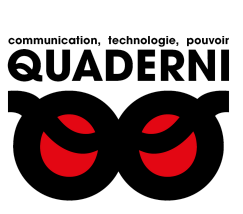

\title{
Quaderni
}

Communication, technologies, pouvoir

95 | Hiver 2017-2018

Logiques numériques des radicalisations

\section{Fictionnalisation des attentats et théorie du complot chez les adolescents}

\section{Vincenzo Cicchelli et Sylvie Octobre}

\section{(2) OpenEdition \\ 1 Journals}

Édition électronique

URL : https://journals.openedition.org/quaderni/1140

DOI : 10.4000/quaderni. 1140

ISSN : 2105-2956

Éditeur

Les éditions de la Maison des sciences de l'Homme

Édition imprimée

Date de publication : 5 février 2018

Pagination : 53-64

Référence électronique

Vincenzo Cicchelli et Sylvie Octobre, «Fictionnalisation des attentats et théorie du complot chez les adolescents », Quaderni [En ligne], 95 | Hiver 2017-2018, mis en ligne le 05 février 2020, consulté le 03 septembre 2021. URL : http://journals.openedition.org/quaderni/1140 ; DOI : https://doi.org/10.4000/ quaderni. 1140 


\section{$D$ ossier}

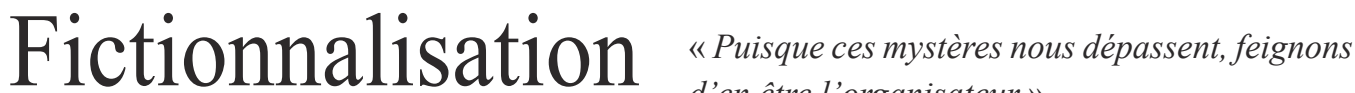 d'en être l'organisateur » des attentats Jean Cocteau, Antigone}

et théorie du

complot chez

Bernard Williams (2002) a mis en évidence deux aspects caractérisant le régime de vérité des sociétés contemporaines (Engel et Rorty, 2005). On observe une très forte méfiance à l'égard de ce qui depuis le siècle des Lumières renvoie aux valeurs de rationalité, de progrès et les adolescents d'objectivité scientifiques, y compris dans des cercles intellectuels, diplômés, dans les médias, dans la classe politique et au sein même des universités. Parallèlement jamais n'a été aussi forte l'impression d'être trompé par les institutions

Vincenzo Cicchelli

Maître de conférence Univesité Paris Descartes Chercheur GEMASS, Paris Sorbonne CNRS

Sylvie Octobre

Chargée de recherche DEP - ministère de la Culture

Chercheure associée GEMASS, Paris Sorbonne CNRS (politiques, scientifiques) qui sont dépositaires de ces valeurs, et jamais les individus n'ont exprimé un tel besoin de confiance systémique (Giddens, 1994) dans un monde gouverné par les risques globaux et fondé sur leur nécessaire anticipation par des savoirs experts (Beck, 2001).

Autrefois réservé à l'exercice de la critique par les philosophes ${ }^{1}$, notamment à l'égard de la religion, des idéologies et du pouvoir, la logique du soupçon s'est largement répandue et porte aujourd'hui également sur la capacité des experts, des journalistes, politiques à produire des vérités objectives, au service du bien-être des citoyens dans une logique de transparence et dans l'intérêt commun. Les rapports aux médias sont ainsi marqués par une distance voire une méfiance généralisée qui s'accompagne de la prolifération de régimes de vérités multiples : fake news ${ }^{2}$, faits alternatifs ${ }^{3}$, hoaxes ${ }^{4}$ ou encore post-vérités ${ }^{5}$. On observe ces tendances tout particulièrement chez les adolescents fortement impliqués dans les usages du numérique et des 
réseaux et nourris aux médias-cultures (Macé et Maigret, 2005 ; Cicchelli et Octobre, à paraître). Comme on le verra dans cet article, c'est dans ce nouveau rapport aux médias-cultures et au numérique qu'il faut alors trouver l'origine, chez les adolescents, de l'insistance sur la nécessité de se forger un esprit critique $e t$ - aussi paradoxal que cela puisse paraitre - de leur sensibilité aux théories du complot (Renard, 2015 ; Mancosu, Vassallo et Vezzoni, 2017).

Notre enquête auprès de lycéens d'établissements des académies d'Aix-Marseille, Créteil, Dijon et Lille fournit des éléments pour comprendre les articulations entre l'omniprésence des imaginaires juvéniles issus des industries culturelles globalisées (musique, cinéma, Internet, etc.), la concurrence des vérités dans un régime de dérégulation de l'information et le recours aux théories du complot pour expliquer les attentats de 2015 et 2016 . Nous partons de l'hypothèse que les imaginaires culturels issus des médias-cultures fournissent des ressources narratives permettant de mettre le monde en récit, ce qui favorise à la fois une perméabilité aux vérités alternatives et le recours à des modes de raisonnement de type complotiste. S'il est souvent admis que la production culturelle a accoutumé nos contemporains à interpréter, décrypter, démasquer (Citton, 2010) - ce qui finit par rendre plus poreuse la frontière entre faits et fictions, vérité et mensonge (Lavocat, 2016, Jameson, 2007 ; Karbovnik, 2015) -, peu d'enquêtes empiriques s'attachent à montrer les contours de cette posture interprétative et soupçonneuse. Encore moins nombreux sont les travaux qui explorent le lien entre cette dernière et une propension chez les individus à adhérer aux théories du complot, à donner une trame narrative à des évènements marquants et exceptionnels tels des attentats terroristes. Nous considérons donc dans cet article les adolescents comme des narratologues ordinaires, c'est-à-dire à la fois comme des interprètes des narrations qui leurs sont proposées et des narrateurs eux-mêmes. Nous tentons de décrire les mécanismes de fictionnalisation par lesquels ces derniers retrouvent dans le réel et dans les médias-cultures des schèmes interprétatifs communs : la fiction est alors un étalon de mesure de la réalité. C'est sur la production de récits personnels d'attentats terroristes au moyen de ressources narratives issues de la fiction que prend appui la revendication de l'esprit critique et du doute hyperbolique chez les adolescents ${ }^{6}$.

\section{La fictionnalisation des attentats : "C'est parce qu'on a vu trop de films qu'on pense comme ça ${ }^{7}$}

Les médias-cultures fournissent aux adolescents des ressources pour produire des récits pluriels, voire antagonistes, du monde. Nous empruntons à Umberto Eco (1996) l'idée que non seulement la fiction a une valeur thérapeutique, mais que la réalité peut être «décortiquée » à l'aide de la fiction. Cette mise en récit du monde par les ressources cognitives et émotionnelles de la fiction est possible dans la mesure où une œuvre fournit une stabilité, une finitude et une vérité que la réalité contemporaine semble incapable de proposer. Les œuvres fictionnelles «nous donnent le sentiment confortable de vivre dans un monde où la notion de vériténe peut être remise en question, alors que le monde réel semble beaucoup plus insidieux» (Eco, 1996, p. 153-154). La prégnance des références média-culturelles dans l'analyse herméneutique du réel est attestée dans des cas 
aussi variés que la réaction à des enlèvements célèbres - dans lesquels les références au cinéma et au roman policier américain ont été importantes (Pinker, 2017) - ou la montée en puissance des lectures complotistes de l'action de l'État-Nation - dans laquelle le succès du roman d'espionnage et du roman policier ont joué un rôle (Boltanski, 2012).

Ainsi dans les entretiens, les adolescents font appel à des références issues des œuvres contemporaines de fiction - notamment les films et les séries-télé - pour répondre au doute créé par ce qui leur semble «incohérent ", "bizarre » ou "pas vrai » dans les informations auxquelles ils ont été confrontés après les attentats. Dans leurs esprits, la réalité ne doit pas laisser place au hasard, pour que l'acteur/spectateur adhère à la cohérence du récit.

Les œuvres de fiction leur fournissent plusieurs repères cognitifs et des logiques narratives (Jost, 2011 ; De Nora, 2014). Tout d'abord, ils fournissent des scansions temporelles qui permettent de se repérer. Ainsi, la date de l'attentat du Bataclan est mémorisée par certains en étant associée à la « finale de Secret Story » (fille, filière générale, académie de Marseille). Ils proposent de surcroît des références contextuelles ou des éléments de décor. Il en va ainsi des prisons, où il faudrait enfermer les auteurs des attentats de 2015 et 2016 qui, selon les adolescents rencontrés, devraient ressembler à Alcatraz, Guantanamo ou aux «prisons mexicaines 》 (garçon, filière pro, académie de Dijon) ou encore à celle de la série télé Prison Break « avec des lits en béton » (garçon, filière pro, académie de Dijon). De même, certains trouveraient normal de recourir à « la torture » ou à « la chaise électrique », qui sont empruntées aux feuilletons et films américains (deux garçons, filière pro, académie de Créteil). Par ailleurs, les fictions regorgent de repères moraux (Ricœur, 1983), notamment à travers des figures de méchants archétypiques, souvent incarnés par des institutions de l'ombre qui manipulent les individus. Ainsi, $50 \%$ des adolescents interrogés estiment que la CIA était impliquée dans les attentats du 11 septembre 2001. Cette figure du " vilain ", on la retrouve sous de nombreuses formes au fil des entretiens : «Parce que ils font plein de trucs bizarres euh... les Américains, toujours!» (fille, filière générale, académie de Marseille). Tel un deus ex machina, le rôle joué par la CIA est souvent mobilisé pour donner du sens aux incohérences supposées du réel. Cela va jusqu'à imaginer au sommet de l'administration américaine des luttes de pouvoir entre le Président et la CIA :

«Mais je pense que la CIA a une main sur le Président, et peut-être que le Président n'a pas fait quelque chose ou a fait quelque chose que la CIA n'a pas aimé et je pense qu'elle a décidé de se venger » (fille, filière générale, académie de Marseille).

Enfin, les fictions proposent des intrigues, mobilisables pour relier des faits, identifier des lieux, des actions, et des personnages et surtout pour tisser des trames d'évènements. Ainsi, il n'est pas rare dans les films d'action qu'un danger imminent soit écarté à la dernière minute - c'est le principe de l'intensité dramatique dénouée par une fin heureuse. Or en appliquant ce principe aux attentats de New York, certains adolescents rencontrés doutent fortement que « des avions remplis de 
passagers se soient vraiment écrasés sur les Tours jumelles » (fille, filière générale, académie de Marseille). Si c'était vrai, selon eux, l'armée américaine serait intervenue avant l'impact et l'avion aurait été intercepté : puisque les avions se sont écrasés, ils étaient évidemment « remplis de chaises vides 》 (garçon, filière générale, académie de Marseille). Ces logiques narratives supposent également toujours l'existence de mobiles, souvent cachés, qu'il faut mettre au jour, puisque le pur hasard ne peut trouver sa place dans l'intrigue fictionnelle. De ce fait, pour certains adolescents, les « Tours jumelles abritaient la réserve fédérale d'or américaine » (garçon, filière pro, académie de Dijon), ce trésor caché justifiant l'attaque, qui, de ce fait, n'est plus (seulement) un attentat mais (aussi) un casse.

Que ces adolescents fassent un recours aussi massif à la fiction pour décortiquer l'actualité n'est pas si surprenant. En effet, les produits culturels de grande audience auprès des adolescents font des références fréquentes aux vérités alternatives, des bandes dessinées (XIII, Histoire secrète, les mangas d'aventure) en passant par des émissions de télévision (Le Vrai Journal), des séries télévisées (Stargate SG-1, Le caméléon, X Files, Utopia), des films (Jason Bourne, Mission Impossible, X-Men, Matrix, etc.), des jeux de société (Les Illuminati), des jeux vidéo (Assassin's Creed, Deus ex Machina), et enfin des livres, comme ceux de David Icke (sur les reptiliens) et les romans de Dan Brown (connus par leurs adaptations cinématographiques). Les consommations culturelles très internationalisées des jeunes (Cicchelli et Octobre, 2017) les habituent à établir des connexions qui elles-mêmes sont planétaires. Ainsi, pour de nombreux ado- lescents interrogés, les attentats de 2015 et 2016 sont directement liés à la position de la CIA dans la guerre de Syrie et/ou à l'interventionnisme de la France en Afrique, tandis que pour d'autres ils impliquent des intérêts financiers mondiaux. Ils construisent une géopolitique de l'ombre censée expliquer l'inexplicable. Cet extrait l'indique :

«Moi je pense que d'un côté, les gens qui font les attentats c'est qu'ils ont une raison de faire ça. Ils sont pas assez fous pour faire des choses comme ça. La France a sûrement dû envoyer certaines personnes pour attaquer leur pays. Ils s'en sont sûrement pris à eux les Français. Et les Syriens, ils ont sûrement dî se venger à leur tour » (garçon, filière pro, académie de Marseille).

\section{Hégémonie des images et incertitude de la vérité : «On ne peut pas savoir»}

La prégnance de ces récits est favorisée par la puissance de l'image animée en diffusion rapide (le verbe d'action le plus employé dans les entretiens au sujet des informations sur les attentats est « voir »). Cette phrase célèbre de Baudrillard - « ce que nous donnent les communications de masse cen'est pas la réalité, c'est le vertige de la réalité » (1970, p. 32) - est encore plus vraie aujourd'hui grâce à la prolifération des images.

L'image donne l'illusion du reflet du réel, de "la preuve par le direct» dont parlent les jeunes, bref elle a valeur de témoignage et il n'est pas étonnant que les médias mentionnés par les adolescents pour s'informer soient les chaînes en continu (BFM, I-Télé...) (Citton, 2010). La force des images est telle qu'elle 
peut convaincre, à l'encontre de toutes opinions majoritaires ou officielles. Leur viralité interdit le temps de la vérification, favorise la réaction pulsionnelle, dans une logique de concurrence, voire de surenchère, entre médias officiels et sources alternatives. La prolifération des images ainsi que celle des émotions produites induisent une incertitude principielle : quasiment tous les adolescents déclarent ne pas pouvoir être sûrs de la véracité de l'information. C'est ce qu'affirme cette jeune fille :

« Je sais pas genre par exemple, des fois sur Internet, ils disent n'importe quoi. Et tandis que sur la télé, ils disent pas la même chose. Mais comme sur la télé des fois, on n'a pas de preuves sûres, sûres, sûres, et après du coup on sait pas s'ils sont faux à la télé ou Internet. Donc du coup, on peut pas savoir » (fille, filière générale, académie de Marseille).

Si les adolescents expriment des doutes concernant la volonté des médias officiels à restituer une information « neutre », c'est tout de même vers eux, télé en tête, qu'ils se tournent au moment des attentats. Dans ce contexte, les réseaux sociaux apparaissent comme un pis-aller néanmoins nécessaire : à la fois moins fiables ( «ils mentent», « on trouve n'importe quoi ») et plus ouverts à la diversité des opinions ("tout le monde peut s'exprimer »). Ainsi, 48\% des adolescents font d'abord confiance aux médias télévisuels français contre seulement $11 \%$ aux blogs et réseaux français. Dans un autre entretien de groupe, des adolescents de filière générale dans l'académie de Marseille le confirment :

Fille : Si, d'un côté c'est bien parce qu'en fait les médias quand ils disent par exemple que c'est Daesh qui a fait l'attentat, ben on n'a pas de preuve que ça soit Daesh ou pas. Donc du coup, quand tu montres des vidéos de Daesh en train de revendiquer l'attentat, on...

Garçon: Ils sont plus crédibles.

Fille: Ils sont plus crédibles. Et on peut mieux les croire. Mais après d'un côté, c'est pareil parce que... je sais pas... après des fois, il se passe des trucs bizarres à la télévision, par exemple ils les égorgeaient ou quoi, mais s'il y a des petits qui sont devant la télé, surtout si c'est sur TF1 ou quoi, ben c'est pas bien de les montrer. Ça a des bons côtés et des mauvais côtés.

(...)

Fille : Mais après, on sait pas. Y'a toujours un doute. Même... on montre des vidéos ou quoi... on sait pas qui est derrière la vidéo. Donc du coup, on sait pas si c'est vraiment eux ou pas. Si c'est un truc inventé par les médias, si c'est un truc inventé par Daesh, on sait pas.

Toutes les enquêtes le montrent (Octobre, 2014), la lecture comme source d'information chez ces adolescents est quasiment absente, mais elle reste, dans leurs représentations, le lieu de l'autorité de la vérité et de la prise de recul (15\% des adolescents déclarent qu'ils font d'abord confiance aux livres et documents écrits), ainsi que l'indique ce jeune garçon :

« Ouais, parce qu'en fait la télé, elle joue sur l'émotion de la personne qui est en train de regarder, tandis que les journaux, ils font un peu plus réfléchir parce que c'est de l'écrit. Donc du coup c'est les médias télévisés qui font, pardonnez-moi l'expression, qui font chier » (garçon, filière générale, académie de Lille). 
Sur fond de méfiance à l'égard des images transmises par les médias officiels, émerge en revanche l'importance accordée aux témoignages informels, relayés par des anonymes. Ce sont les témoignages qui permettent d'être au plus près de la réalité (avoir vu les attentats, avoir filmé de première main les événements et mettre directement et immédiatement à disposition sur les réseaux sociaux ces vidéos), en faisant donc l'économie des récits produits par les médias. Une jeune fille l'expose ainsi :

«Ben, moi je fais plus confiance aux gens et à ce qu'ils voient parce que moi-même si je verrais quelque chose, ben je me ferais plus confiance que regarder à la télé et voir ce qu'ils me disent, parce que des fois ils déforment les informations et c'est pas forcément vrai. Alors qu'une personne dans la rue, elle a vu la scène, elle est témoin. Elle sait ce qu'elle a vu» (fille, filière pro, académie de Marseille).

\section{Idéal démocratique de l'empowerment par les TIC : "Chacun a droit à avoir son idée personnelle »}

Comment comprendre cette faveur dont jouissent les récits alternatifs et la méfiance à l'égard des récits officiels chez les jeunes (Reichstadt, 2015) ? Le succès des technologies de l'information et de la communication - Internet en tête - est né du mythe démocratique de la participation de tous (Cardon, 2010). Le fonctionnement culturel du numérique est en effet basé sur la participation horizontale et non sur l'expertise et la mise en doute de l'autorité officielle y est la règle. En effet, l'Express Yourself des réseaux sociaux et des nouvelles technologies (Allard et Vanderberghe, 2003 ; Jenkins, 1992 ; 2013) a renforcé l'idée notamment chez les plus jeunes : a) que la légitimité d'une assertion trouve son fondement dans l'individualité de celui ou celle qui l'énonce ; b) que les médias et voix alternatifs peuvent dévoiler la face cachée des choses - le tout dans une prolifération d'informations qui rend impossible l'examen exhaustif des arguments disponibles (Bronner, 2015). Ces deux traits accréditent l'idée que chacun a le droit d'être l'auteur d'un récit dans un espace public nouveau, celui d'Internet et des réseaux sociaux. Un jeune garçon l'exprime en mettant en avant la force des réseaux sociaux dans la contestation des caricatures de Charlie Hebdo :

« en fait dans les réseaux sociaux, il y a des personnes déjà qui se plaignaient de ce qui se passait à Charlie Hebdo, sauf qu'ils (les dessinateurs de Charlie Hebdo) s'en foutaient carrément, ils continuaient » (garçon, filière générale, académie de Lille).

La liberté d'expression (revendiquée comme une valeur dans les entretiens par les jeunes), l'exigence de transparence (également appelée de leurs vœux) et l'idéal de participation (qui est celui des réseaux) favorisent la diffusion simultanée d'informations de nature et de qualité très variables, tandis que la norme du pluralisme des valeurs et des opinions autorise que tout (ou presque) puisse être discuté, par quiconque, de toutes les manières. Ceci peut donner lieu à deux phénomènes (Derrida, 2012) : soit des mensonges (délibérés et intentionnels), soit des altérations sans modèle assuré et sans intentionnalité (qui ne sont pas à proprement parler des mensonges). Cette seconde forme est à la fois 
plus bénigne puisqu'aucune intention organisée de tromper n'existe, et plus dangereuse, car elle altère les normes interprétatives et rend difficile le consensus. Dans notre enquête, près des trois quarts des adolescents déclarent ne pas suivre les opinions des autres et un tiers ne faire confiance qu'à leurs idées personnelles, et l'expression «par moi-même » revient souvent dans les entretiens. À l'extrême, certains adolescents estiment que les vérités alternatives sont la marque de la liberté individuelle : " le complot c'est des gens libres qui s'entraident, enfin qui s'entraident, qui s'entraident euh... pff, pour faire des trucs cools ou pas cools » (garçon, filière générale, académie de Dijon). Mais les reprises d'énoncés en cascade peuvent aussi générer un effet global de dé-réalité : dans les entretiens, les questions «c'est quoi le réel? ? et «c'est quoi la vérité?» sont sans cesse présentes.

Reste que, les adolescents que nous avons rencontrés, qu'ils soient croyants ou pas, quelles que soient leurs origines, et quelles que soient leurs filières scolaires, ont exprimé une - et une seulelimite : le respect de la croyance. «On ne peut pas rire de tout » est un jugement quasi unanime dans les entretiens et dans l'enquête quantitative, $84 \%$ des adolescents rejettent l'idée qu'on puisse se moquer de tout y compris des religions. Cela distingue fortement le registre religieux, devenu aujourd'hui identitaire dans le double sens individuel et collectif, des opinions politiques.

\section{Esprit critique et théorie du complot : "Appa- remment, il y a des trucs cachés "}

La prolifération de vérités alternatives est liée à l'imaginaire démocratique contemporain
(Girardet, 1986) et exprime un délire sur le pouvoir, notamment en niant la part de hasard du réel et en surinterprétant les faits (Taïeb, 2010). Pour comprendre cette incertitude de la vérité (Nicolas, 2014), il faut revenir sur la nature des idées alternatives ou complotistes qui, comme le rappelle Gérard Bronner (2016), ne sont pas irrationnelles. Leur développement est directement lié aux progrès de la connaissance dans la mesure où celui-ci accroît la conscience de l'inconnu et l'inconfort qui en découle, tout en mettant à disposition des adolescents, à travers l'éducation reçue au collège et au lycée, les linéaments de l'esprit critique. Ainsi, en aiguisant le sens critique, l'éducation rend les vérités moins définitives.

Le paradoxe de l'éducation dans son apport à la diffusion d'une culture du soupçon trouve son équivalent dans le paradoxe des médias cultures. Le cadre des médias culture et de la place qu'y tient la fictionnalisation donne corps à une nouvelle conception de «l'esprit critique » entendu moins comme aptitude à tenir une proposition pour vraie ou fausse en la passant au crible de la raison que comme une réception des médias se fondant sur une posture interprétative, sur des actes de braconnage, de picorage et de réagencement - cette posture devenant un mode d'expression de l'individualité (Flichy, 2010 ; Jenkins, 2013). L'extrait suivant illustre comment raisonnement logique et appel à l'opinion personnelle cohabitent de façon paradoxale :

« Je sais pas, je pense qu'il y a une manipulation mondiale, quelque chose de gros, mais plus c'est gros, plus ça passe. (...) Ça veut dire que pff... deux avions détournés comme ça, dans deux tours, le Pentagone aussi, qu'un avion est 
soi-disant rentré dedans. Je sais pas, c'est pas un trou de Boeing qu'il y avait dedans. Il faisait trois mètres de haut le trou, ils ont pas retrouvé d'images d'avion. Ils ont trouvé la CIA avait tout pris. Il y a les avions, ils ont retrouvé qu'une image seconde par seconde puis on voit rien et puis après on voit que ça a pété quoi. Des trucs comme ça, mais... (...) Ben, il y aurait peut-être une manipulation mondiale, mais qu'on n'est pas au courant, parce qu'on est directement dedans, c'est un truc que j'ai conclu moi-même ça»" (garçon, filière pro, académie de Dijon).

Le discours de ce jeune garçon emprunte en effet au complotisme quatre éléments typiques - le doute systématique quant aux faits avérés par les investigations menées par les autorités, l'importance accordée au moindre détail et notamment ceux passés sous silence dans les versions officielles (Keeley 1999), la recherche d'explications alternatives, l'identification d'acteurs et de groupes qui manœuvrent en coulisse - et s'arrime sur l'idée d'une conclusion personnelle.

Reste que les conceptions que les adolescents ont de l'esprit critique, y compris chez ceux qui ne sont pas complotistes, sont très variables et assez éloignées de la démarche critique à laquelle ils prétendent s'adosser. On peut discerner plusieurs critères de mise en œuvre de l'esprit critique dans les entretiens chez certains adolescents :

a) la confrontation des sources, affirmée par principe mais rare en réalité : il s'agit de «multiplier les sources 》, «discuter », " décortiquer 》, et/ ou de se référer à des médiateurs (souvent les parents) ;

b) le jugement de vraisemblance : le fait que la carte d'identité d'un des deux auteurs de l'attentat de Charlie Hebdo ait été retrouvée dans leur voiture provoque chez les adolescents un doute sur la véracité de la version officielle, puisque la probabilité qu'un terroriste laisse des traces aussi évidentes de son identité est pour eux $a$ priori faible ;

c) le jugement sur l'autorité de l'énonciateur : les adolescents reconnaissent au métier de journaliste la compétence de traitement de l'information, ce qu'ils ne reconnaissent pas aux blogueurs.

Mais ces critères sont parfois contradictoires : si le journaliste de métier est a priori plus crédible, reste que $50 \%$ des adolescents ne font pas confiance aux médias. De même, ils reconnaissent l'importance du métier de journalistes, mais ils déclarent privilégier les témoins ordinaires directs qui postent des vidéos qu'ils ont euxmêmes tournées, les considérant comme plus « authentiques » que les informations issues des médias. La situation d'incertitude est donc prorogée.

\section{Conclusion}

La faveur dont jouissent les vérités alternatives est la face cachée et paradoxale tant du succès du modèle démocratique d'accès à et de production de l'information que du rôle des médias cultures dans l'éducation buissonnière des jeunes (Barrère, 2011). Très grands consommateurs de produits des médias-cultures globalisées, les jeunes sont entourés de fictions (Lavocat, 2016), et développent des compétences de narratologues ordinaires. Ainsi qu'Umberto Eco l'indiquait dans l'ouvrage Six promenades dans les bois du roman et d'ailleurs (1996, p. 153-154), «Les 
crypto-analystes ou les décrypteurs de code secret obéissent à une règle d'or : tout message est déchiffrable pourvu que l'on sache qu'il s'agit d'un message. Avec le monde réel, le problème est que nous nous demandons depuis la nuit des temps si ce message existe et s'il a un sens. Quant à l'univers narratif, nous avons la certitude qu'il constitue un message, qu'une autorité d'auteur est derrière lui, comme étant son origine et un ensemble d'instructions pour la lecture ». Le lecteur d'Eco s'est mué en spectateur de séries télé et de films et en consommateur de vidéos sur Internet. Armés de ces ressources narratives, les adolescents - y compris complotistes - tentent de reprendre le pouvoir face aux difficultés qu'ils affrontent pour lire le monde, à mettre de la cohérence là où il ne semble pas y en avoir.

\section{$R \cdot E ́ E \cdot F \cdot E \cdot R \cdot E \cdot N \cdot C \cdot E \cdot S$}

APPADURAI A., Géographie de la colère. La violence à l'ère de la globalisation, Paris, Payot, 2006.

ALLARD L. et VANDENBERGHE F., « Express yourself ! Les pages perso. Entre légitimation technopolitique de l'individualisme expressif et authenticité réflexive peer to peer ", Réseaux, 2003/1 (no 117), p 191-219.

BARRÈRE A., L'éducation buissonnière. Quand les adolescents se forment par eux mêmes, Paris, Armand Colin, 2011.

BAUDRILLARD J., La société de consommation, Paris, Gallimard, 1970.

BECK U., La société du risque, Paris, Aubier, 2001.

BRONNER G., La pensée extrême. Comment des hommes ordinaires deviennent des fanatiques,

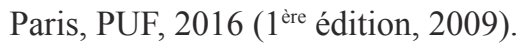

BOLTANSKI L., Énigmes et complots : Une enquête à propos d'enquêtes, Paris, Gallimard, 2012.

CARDON D., La démocratie Internet, Paris, Seuil, 2011.

CICCHELLI V. et OCTOBRE S., L'amateur cosmopolite. Goûts et imaginaires juvéniles à l'ère de la globalisation, Paris, MCC, 2017.

CICCHELLI V. et OCTOBRE S., « La radicalité informationnelle : prolifération des vérités alternatives, défiance à l'égard des médias et théorie du complot », in O. Galland et A. Muxel (dir), $L a$ tentation radicale. Enquête auprès des lycéens, Paris, PUF, (à paraitre en 2018).

CITTON Y., L'avenir des humanités. Économie de la connaissance ou cultures de l'interprétation?, Paris, La découverte, 2010. 
DENORA T., Making sense of reality, culture and perception in everyday life, Sage, 2014.

DERRIDA J., Histoire du mensonge, Paris, Galilée, 2012.

ECO U., Six promenades dans les bois du roman et d'ailleurs, Paris, Grasset, 1996.

ENGEL P. et RORTY R., À quoi bon la vérité? Paris, Grasset, 2005.

GALLAND O. et MUXEL A. (dir), La tentation radicale. Enquête auprès des lycéens, Paris, PUF, (à paraitre en 2018).

GIDDENS A., Les conséquences de la modernité, Paris, L'Harmattan, 1994.

JAMESON F., La totalité comme complot, Conspiration et paranoïa dans l'imaginaire Contemporain, Paris, Les Prairies Ordinaires, 2007.

JENKINS H., Textual Poacher, New York, Routledge, 2002.

JENKINS H., La culture de la convergence, Des medias au transmédia, Paris, Armand Colin, 2013.

JOST F., De quoi les séries américaines sont-elles le symptôme? Paris, CNRS Èditions, 2011.

KARBOVNIK D., "Théorie du complot et OVNIS », Diogène, n² 249-250, 2015, p. 240251.

MAIGRET E. et MACÉ E., Penser les mediacultures. Paris, Colin, 2005.

LAVOCAT F., Fait et fiction. Pour une frontière, Paris, Seuil, 2016.

MANCOSU M., VASSALLO S. et VEZZONI C., Prevalence and Determinants of Conspiracy Theories in Italy. Evidence from an exploratory analysis on survey data, WP, 2017. (https://www. researchgate.net/publication/316457096).

NICOLAS L., «L'évidence du complot : un défi à l'argumentation. Douter de tout pour ne plus douter du tout », Argumentation et analyse du discours, [En ligne], 13 | 2014, mis en ligne le 14 octobre 2014, consulté le 19 octobre 2017. URL : http://aad.revues.org/1833 ; DOI : 10.4000/ aad.1833.

PINKER R., Faire sensation. De l'enlèvement du bébé Lindbergh au barnum médiatique, Marseille, Agone, 2017.

RENARD J.-B., « Les causes de l'adhésion aux théories du complot », Diogène, n 249-250, 2015, p. 107-119.

REICHSTADT R., « Conspirationnisme : un état des lieux », Note de la Fondation Jean Jaurès - Observatoire des radicalités politiques, 24/02/2015 < http://temps-reels.net/ site/wp-content/uploads/2015/04/2015-02-24_ FJJ_conspirationnisme.pdf $>$

RICEUR P., De l'interprétation. Essai sur Sigmund Freud, Paris, Seuil, 1965.

RICCEUR P., Temps et récit. I : L'intrigue et le récit historique, Paris, Seuil, 1983.

TAÏEB E., « Logiques politiques du conspirationnisme », in V. Tournay (dir.), Sociologie et Sociétés, «Quand le vivant devient politique », 42-2, 2010, p. 265-289.

WILliAMS B., Truth and Truthfulness, Princeton, Princeton University Press, 2002 (trad. Vérité et véracité, Paris, Gallimard, 2006). 


\section{$N \cdot O \cdot T \cdot E \cdot S R \cdot E \cdot S \cdot U \cdot M \cdot E$}

1. Dans son essai célèbre sur l'interprétation, Paul Ricoeur (1965) ne désigne-t-il pas Marx, Nietzsche et Freud comme les «maîtres du soupçon »?

2. Information fausse qui contrefait un article de presse. Cf. sur ce point l'enquête menée par $L e$ Monde sur Facebook:http://www.lemonde.fr/lesdecodeurs/article/2017/07/05/enquete-sur-lesusines-a-fausses-informations-qui-fleurissentsur-facebook_5156313_4355770.html

3. Concept inventé par Kellyanne Conway, conseillère à la Maison-Blanche de Donald Trump lors d'une interview sur NBC le 22 janvier 2017. Le fait alternatif est une contre-vérité.

4. Canular viral, souvent diffusé par e-mail.

5. Théorie selon laquelle l'émotion et la croyance comptent désormais plus que les faits avérés.

6. Cette recherche, dirigée par Olivier Galland et Anne Muxel, s'inscrit dans le cadre de l'appel à projets lancé par le président du CNRS Attentats-Recherches - au lendemain des attaques du Bataclan le 13 novembre 2015. Elle a été réalisée dans 4 académies (Créteil, Marseille, Dijon et Lille) auprès de jeunes de classes de seconde, par entretiens et par questionnaires. Voir Galland et Muxel (dir) (à paraître).

7. Tous les éléments présentés en italiques sont des verbatim.
S'il est admis que la production culturelle a accoutumé les adolescents à endosser une posture interprétative, peu d'enquêtes s'attachent à en montrer les contours et à explorer le lien entre cette dernière et une propension à adhérer aux théories du complot. Nous considérons ici les adolescents comme des narratologues ordinaires et tentons de décrire les mécanismes de fictionnalisation par lesquels ces derniers retrouvent dans le réel et dans les médias-cultures des schèmes interprétatifs communs.

\begin{abstract}
Number of research have shown how cultural products require from teenagers to assume an interpretative stance, but researches seldom highlight the contours of this interpretative work, and its links with conspiracy theories. In this article, we consider teenagers as ordinary narratologists, and try to analyze the fictionalisation mechanisms, through which young people find common interpretative schemes in real life and media-cultures.
\end{abstract}


